\title{
Effects of Structure Parameters on Temperature Characteristics of Multiple Quantum Well Ring Laser
}

\author{
Jing Guo \\ School of Electronic Information Engineer \\ Tianjin University \\ Tianjin, China \\ e-mail: yanyuan_guoguo@163.com
}

\author{
Sheng Xie \\ School of Electronic Information Engineer \\ Tianjin University \\ Tianjin, China \\ e-mail: xie_sheng06@tju.edu.cn
}

\author{
Hao Wang \\ School of Electronic Information Engineer \\ Tianjin University \\ Tianjin, China \\ Weilian Guo \\ School of Electronic Information Engineer \\ Tianjin University \\ Tianjin, China
}

\begin{abstract}
Due to the potential application in all-optical signal processing, semiconductor ring laser (SRL) has become one of research hotspots in the present. In this paper, an equivalent SRL was modeled utilizing a numerical device simulator (ATLAS), and the effects of structure parameter on the temperature characteristics of multiple quantum well SRL were simulated and analyzed with temperature range from $260 \mathrm{~K}$ to $460 \mathrm{~K}$. The simulation results indicated that an optimal quantum well structure with well width around $20 \mathrm{~nm}$ and well number of five existed for the lowest threshold current density. And the ring radius has little effect on the temperature characteristics of differential quantum efficiency and lasing wavelength.
\end{abstract}

Keywords- temperature characteristics; semiconductor ring laser; multiple quantum well; gallium arsenide

\section{INTRODUCTION}

With the rapid development of optical fiber transmission technology, High-speed signal processing capacity at the switching nodes in optical communication system is required. However, due to the limitation of electronic bottleneck, conventional electronic switching scheme is increasingly challenged. To realize high-speed optical communication network, it is therefore necessary to substitute the electronic router with all-optical signal processing system by integrating the photonic devices in one chip. At present, various implementation schemes have been proposed and employed, such as semiconductor optical amplifier-based Mach-Zehnder interferometer $(\mathrm{SOI}-\mathrm{MZI})^{[1]}$, nonlinear optical loop mirror $(\mathrm{NOLM})^{[2]}$, semiconductor ring laser (SRL) ${ }^{[3]}$, and so on. Compared with the other photonic devices, semiconductor ring lasers (SRLs) have received increasing attention, because they can be fabricated without the need for cleaved facet or gratings for optical feedback ${ }^{[4]}$. Moreover, SRLs have the benefits of compact structure, high density integration, optical bistability, and excellent wavelength selectivity ${ }^{[5]}$. These features make SRLs particularly suitable for the large scale photonic integrated circuit. Therefore, SRLs are increasingly seen as a promising approach for integrated all-optical functional devices.

Up to now, many excellent review articles and research reports on device fabrication and characterization has been published $^{[6,7]}$, and SRLs have been widely applied in the field of all-optical signal processing, such as optical memory ${ }^{[8]}$, all-optical logic gate ${ }^{[9]}$, and label swapping ${ }^{[10]}$. As we know, the device parameter of SRL, such as threshold current, differential quantum efficiency, and lasing wavelength, varies accordingly with the operating temperature. However, this temperature dependence seriously affects the stability and reliability of SRLs, and brings about many problems in application. Therefore, the temperature characteristic of SRL is an important issue for the device application. To improve the temperature stability, optimizing the structure parameters of SRL device is necessary.

In this paper, we firstly analyze the temperature dependence of the key parameters for multiple quantum well SRL. And an equivalent SRL is modeled utilizing a numerical device simulator (ATLAS). The effects of variation of the device structural parameters, such as ring radius, well width and well number, on the temperature characteristics of SRL are simulated and analyzed with temperature range from $260 \mathrm{~K}$ to $460 \mathrm{~K}$.

\section{DEVICE STRUCTURE AND THEORETICAL ANALYSIS}

The schematic illustration of a typical SRL, with a straight waveguide coupled to the ring resonator, is shown in Fig.1. The gap between the ring cavity and the straight waveguide is $1 \mu \mathrm{m}$, providing a theoretical amplitude coupling ratio of $1 \%-5 \%$ depending on the ring radius and the etching depth of the waveguides.

We suppose the SRL devices are fabricated in standard GaAs/AlGaAs multiple quantum well (MQW) material system. The active region has several GaAs quantum wells separated by $\mathrm{Al}_{0.5} \mathrm{Ga}_{0.5} \mathrm{As}$ barriers. The p-type and n-type cladding layers consist of $1.0 \mu \mathrm{m}$-thick $\mathrm{Al}_{0.3} \mathrm{Ga}_{0.7} \mathrm{As}$ and 2.0 $\mu$ m-thick $\mathrm{Al}_{0.5} \mathrm{Ga}_{0.5} \mathrm{As}$, respectively. The cross section of MQW ridge waveguide is depicted in Fig. 2, where the 
waveguide width and the etching depth are $1.0 \mu \mathrm{m}$ and $0.9 \mu \mathrm{m}$, respectively.

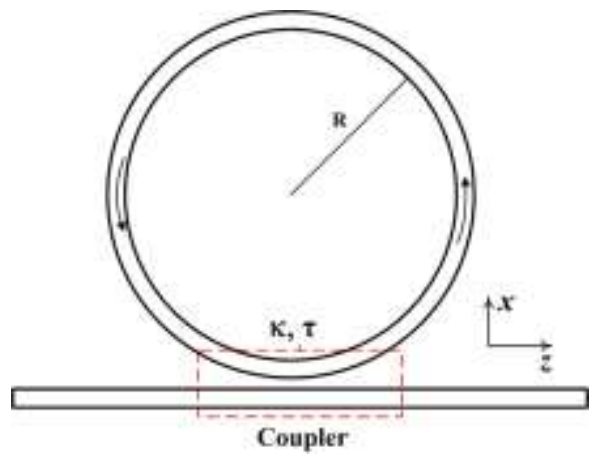

Figure 1. Schematic illustration of a typical SRL

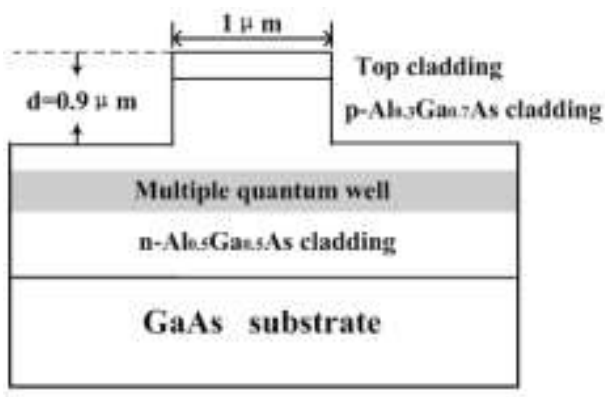

Figure 2. Cross-section of MWQ ridge waveguide

Based on the laser oscillation condition of a FabryPerot cavity ${ }^{[11]}$, the threshold gain for the circular SRL as shown in Fig. 1 can be modified as

$$
T \exp \left[\left(g-\alpha_{w}\right) L\right] \exp \left[\frac{i 2 \pi n_{\text {eff }} L}{\lambda}\right]=1
$$

Where $T=1-\kappa^{2}$ represents the power transmission ratio of the coupler, $\kappa$ is the amplitude coupling ratio, $g$ is the material gain, $\alpha_{\mathrm{w}}=\alpha_{i}+\alpha_{b}+\alpha_{s c}$ is the loss of ring cavity, $\alpha_{i}$ is the intrinsic waveguide loss, $\alpha_{b}$ represents the bending loss, which is a function of the ring radius and the etching depth, $\alpha_{s c}$ represents the scattering loss caused by ridge waveguide sidewall roughness, $L=2 \pi R$ is the cavity length, $R$ is the ring radius, $n_{\text {eff }}$ is the effective index of ring cavity, $\lambda$ is the vacuum wavelength.

From equation (1), the resonance condition and the gain condition of a circular SRL can be written as

$$
\begin{gathered}
m \lambda=n_{\text {eff }} \cdot L \\
T \exp \left[\left(g-\alpha_{w}\right) L\right]=1
\end{gathered}
$$

Where $m$ is an integer.

When the wavelengths satisfy the resonance condition, they will be lased and output the straight waveguide. Experimental results found that the temperature shift of lasing wavelength in the quantum well semiconductor lasers can be described as following ${ }^{[12]}$

$$
\frac{d \lambda}{d T}=a_{1}-\left(a_{2} / T_{0}\right)
$$

Where $a_{1}$ is a red-shift parameter in wavelength with temperature at constant current, and $a_{2}$ is a blue-shift parameters in wavelength with current at constant temperature, and they are not depend on the device structure. $T_{0}$ represents the characteristic temperature.
Considering the gain saturation at a high injection level, the gain of MQW circular laser can be well approximated by the well-known logarithmic form ${ }^{[13]}$

$$
g=g_{0}\left[1+\ln \left(\frac{J}{J_{0}}\right)\right]
$$

Where $g_{0}$ is the gain saturation parameter, $J_{0}$ is the transparency current density.

If we neglect the coupling between the quantum wells, the optical confinement factor of MQW can be approximated as $M$ times of the single quantum well. Therefore, the modal material gain of MQW ring laser is given by $g_{m}=M \Gamma_{w} g_{w}$. Where $M$ is the number of quantum well, $\Gamma_{w}$ is the optical confinement factor of one well, $g_{w}$ is the material gain per quantum well.

At threshold,

$$
M \Gamma_{w} g_{w, h}=\alpha_{w}+\alpha_{c}
$$

is satisfied. Where $\alpha_{c}$ is the coupling loss. For the considered circular laser, it is defined as

$$
\alpha_{c}=-\ln \left(1-\kappa^{2}\right) / L
$$

Combining equations (5)-(7), the threshold current density can be derived as

$$
J_{t h}=J_{0} \exp \left(\frac{\alpha_{w}-\ln \left(1-\kappa^{2}\right) / L}{M \Gamma_{w} g_{0}}-1\right)
$$

The temperature dependence of $J_{t h}$, based on empirical observations, was described by ${ }^{[14]}$

$$
J_{t h}=J_{t h}\left(T_{r}\right) \exp \left(\frac{T-T_{r}}{T_{0}}\right)
$$

Where $T_{r}$ is room temperature.

As a key parameter of ring lasers, the differential quantum efficiency $\eta_{d}$, which represents the conversion efficiency between light output power and injection current, is given by

$$
\eta_{d}=\eta_{i} \frac{\alpha_{w}}{\alpha_{w}+\alpha_{c}}
$$

Where $\eta_{i}$ is the internal quantum efficiency.

Assuming $\eta_{i}$ is constant, the temperature dependence of $\eta_{d}$ is approximated as

$$
\frac{d}{d T}\left(\frac{1}{\eta_{d}}\right)=\frac{1}{\eta_{i} \alpha_{c}} \frac{d \alpha_{w}}{d T}=\left(\frac{L}{\eta_{i} \ln \left(1-\kappa^{2}\right)^{-1 / 2}}\right) \frac{d \alpha_{w}}{d T}
$$

\section{RESULTS AND DISCUSSION}

Based on the device simulation software (ATLAS), an equivalent SRL was modeled. Here, the free carrier loss and the spontaneous recombination model were adopted. Additionally, the recombination models of Auger, Shockley-Read-Hall, and radiative recombination were specified. Numerical calculations were carried out utilizing Newton's iteration method to investigate the influence of structural parameters on the temperature characteristics of SRL. The key physical parameters adopted in the simulation are summarized in Table 1.

Fig. 3 shows the temperature dependence of the threshold current density with different structural parameters. As can be seen, the threshold current density increases with increasing the temperature. This is due to the increase of transparent carrier density caused by the 
TABLE 1 PHYSICAL PARAMETERS USED IN THE SIMULATION

\begin{tabular}{cll}
\hline Symbol & \multicolumn{1}{c}{ Description } & \multicolumn{1}{c}{ Value } \\
\hline$n_{1}$ & Index of air & 1.0 \\
$n_{2}$ & Index of core & 3.5 \\
$n_{3}$ & Index of cladding & 3.17 \\
$\tau_{n 1}$ & Electron lifetime of AlGaAs & $2 \times 10^{-9} \mathrm{~s}$ \\
$\tau_{n 2}$ & Electron lifetime of GaAs & $1 \times 10^{-8} \mathrm{~s}$ \\
$\tau_{p 1}$ & Hole lifetime of AlGaAs & $2 \times 10^{-9} \mathrm{~s}$ \\
$\tau_{p 2}$ & Hole lifetime of GaAs & $1 \times 10^{-8} \mathrm{~s}$ \\
$R c$ & Radiative recombination rate & $1.5 \times 10^{-10}$ \\
$\mu_{n 1}$ & Electron mobility of AlGaAs & $2400 \mathrm{~cm}^{2} / \mathrm{Vs}$ \\
$\mu_{n 2}$ & Electron mobility of GaAs & $4600 \mathrm{~cm}^{2} / \mathrm{Vs}$ \\
$\mu_{p 1}$ & Hole mobility of AlGaAs & $800 \mathrm{~cm}^{2} / \mathrm{Vs}$ \\
$\mu_{p 2}$ & Hole mobility of GaAs & $2500 \mathrm{~cm}^{2} / \mathrm{Vs}$ \\
\hline
\end{tabular}

broadening energy distribution of carrier injection. For a given temperature, the threshold current density increases with the increase of well width and well number, as shown in Fig. 3(a) and (b), but it decreases with increasing of ring radius (shown in Fig. 3(c)). When the radius exceeds
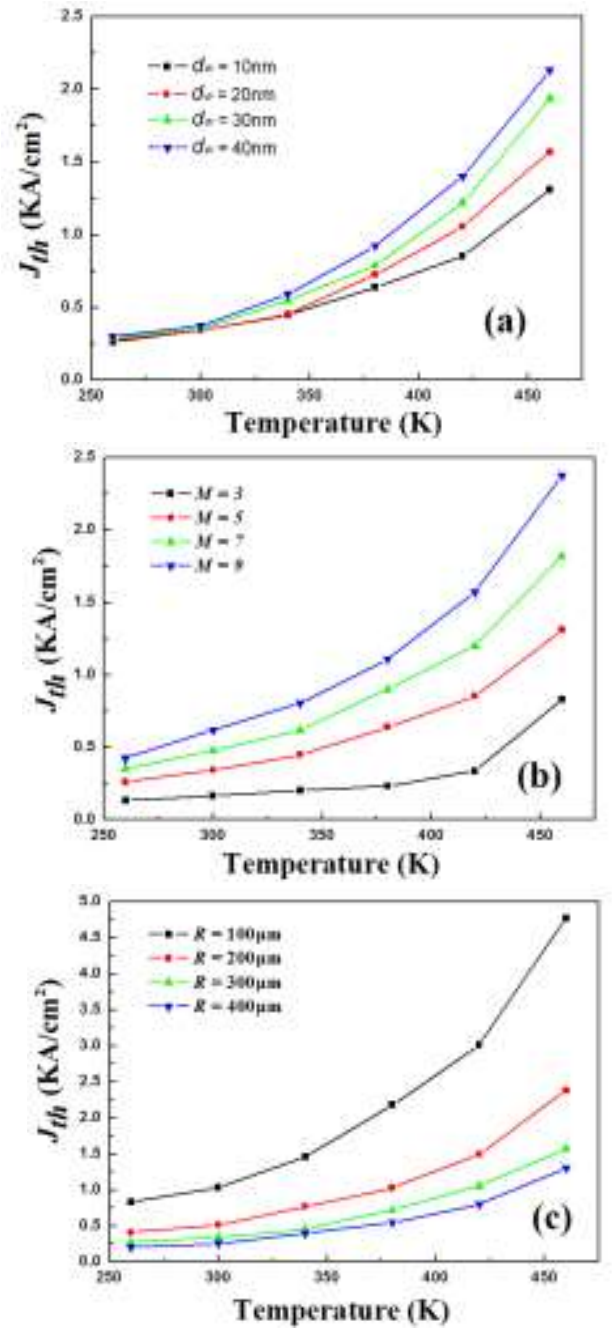

Figure 3. Temperature dependence of threshold current density with different structural parameters. (a) well widths of 10, 20, 30 and 40nm with five-well and $300 \mu \mathrm{m}$ radius; (b) well numbers of 3, 5, 7, 9 with $10 \mathrm{~nm}$ well width and $300 \mu \mathrm{m}$ radius; (c) ring radii of 100, 200, 300, 400 $\mu \mathrm{m}$ with five-well and $20 \mu \mathrm{m}$ well width.
$400 \mu \mathrm{m}$, the effect of radius on the threshold current density becomes small, but oversize radius will decrease free spectral range of ring cavity.

To achieve a lower threshold current density, the dependence of $J_{t h}$ on the well width with a fixed five-well structure was simulated at room temperature, as shown in Fig. 4. For the considered circular laser, the threshold current density decreases with the increase of well width initially, and reaches the lowest value of $1.0 \mathrm{KA} / \mathrm{cm}^{2}$ around well width of $20 \mathrm{~nm}$. When the well width exceeds $20 \mathrm{~nm}$, the threshold current density increases rapidly. This is consistent with the results of MQW semiconductor laser. Therefore, appropriate values of well width and well number need to be selected to obtain the lowest threshold current and minimum temperature dependence.

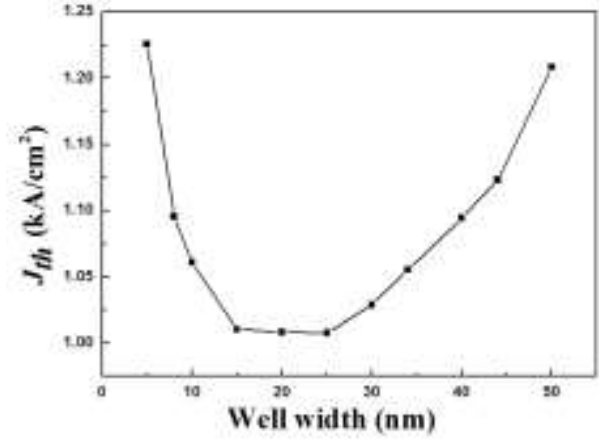

Figure 4. Threshold current density as a function of well width

Since the threshold current is a key parameter affecting the temperature characteristics of ring laser. The optimal well width of $20 \mathrm{~nm}$ and well number of 5 are adopted in the following simulation. Fig.5 shows the differential quantum efficiency as a function of temperature with different ring radii. It is obvious that the differential quantum efficiency decreases with increasing the temperature. This is due to the increasing loss caused by the free carrier absorption and the carrier overflowing from active layer ${ }^{[14]}$. Moreover, the differential quantum efficiency increase slightly with the increase of radius, this is because of the reduced internal loss.

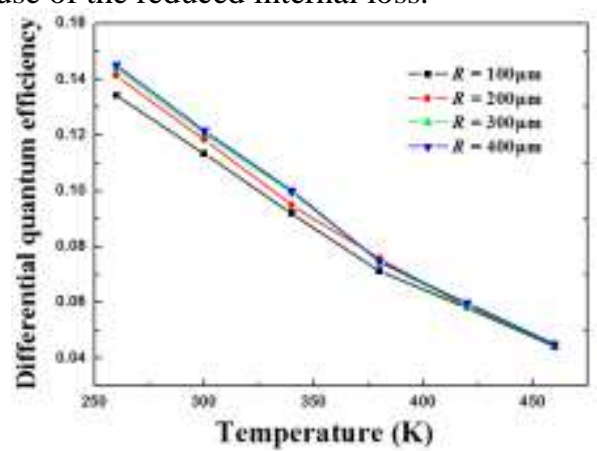

Figure 5. Differential quantum efficiency as a function of temperature with different ring radii.

The lasing wavelength as a function of temperature with different ring radii is plotted in Figure 6. As can be seen from this figure, the lasing wavelength almost increases linearly with increasing temperature, and the effect of ring radius on lasing wavelength can be neglected, which means the temperature dependence of lasing wavelength is almost independent of ring radius. 


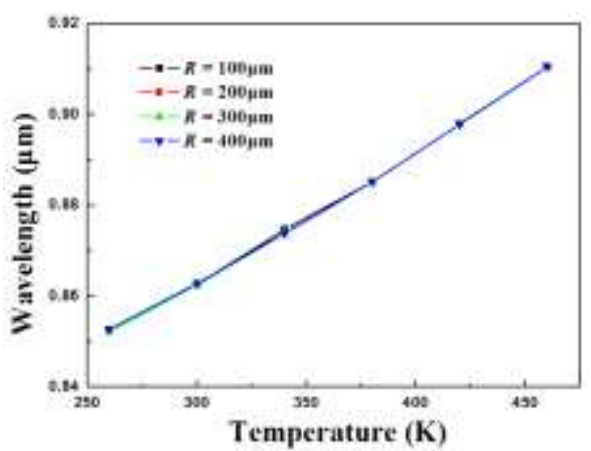

Figure 6. Temperature dependence of lasing wavelength with different ring radii.

\section{CONCLUSION}

The key performance parameters of MQW ring laser and their temperature dependence were analyzed. And an equivalent SRL was modeled utilizing a numerical device simulator (ATLAS). The effects of ring radius, well width and well number on the threshold current density were simulated with temperature range from $260 \mathrm{~K}$ to $460 \mathrm{~K}$, and an optimal quantum well structure with well width of $20 \mathrm{~nm}$ and well number of five was achieved for the lowest threshold current density. Based the optimal material structure, the temperature dependence of the differential quantum efficiency and the lasing wavelength was calculated with different ring radii. Simulation results indicated that the radius has little effect on these two parameters.

\section{ACKNOWLEDGMENT}

The work was supported by the National Natural Science Foundation of China under Grant 61106052.

\section{REFERENCES}

[1] D. K. Gayen, A. Bhattachryya, T. Chattopadhyay, and J. N. Roy, "Ultrafast all-optical half adder using quantum-dot semiconductor optical amplifier-based Mach-Zehnder interferometer," J. Lightw. Technol., vol.30, pp. 3387-3393, Nov. 2012.

[2] D. Williams, X. Bao, and L. Chen, "All-optical NAND/NOT/AND /OR logic gates based on combined brillouin gain and loss in an optical fiber," Appl. Opt., vol.52, pp. 3404-3411, May, 2013.

[3] A. Trita, G. Mezosi, M. Sorel, G. Giuliani, "All-optical toggle flipflop based on monolithic semiconductor ring laser". IEEE Photon. Technol. Lett. vol.26, pp. 96-99, Jan. 2014.

[4] S Xie, W Guo, X Li, L Qi, X Yu, and J Yu, "Design and fabrication of InP-based low threshold microring laser with uniqure unidirectional bistability operation", Chin. J. Laser, vol.38, pp.0302011-1-4, Mar. 2011

[5] T. Segawa, S. Matsuo, T. Kakisuka, Y Shibata, and T. Sato, "Monolithically integrated wavelength-routing switch using tunable wavelength converters with double-ring-resonator tunable Lasers," IEICE Trans. Electron., vol.E94-C, pp. 1439-1446, Sep. 2011.

[6] S. Y. Yu, "All-optical functions based on semiconductor ring lasers," Chin. Opti. Lett., vol.8, pp. 918- 923, Sep. 2010.

[7] M. Sorel, G. Giuliani, A. Scire, R. Miglierina, S. Donati, and P. J. R. Laybourn, "Operating regimes of GaAs-AlGaAs semiconductor ring lasers: experiment and model", IEEE J. Quantum Electron., vol.39, pp.1187-1195, Oct. 2003.

[8] M. T. Hill, H. J. S. Dorren, T. de Vries, J. M. Leijtens, and J. H. den Besten, "A fast low-power optical memory based on coupled micro-ring laser". Nature, vol.432, pp. 206-208, Nov. 2004.

[9] B. Li, M. I. Memon, G. Mezosi, Z. Wang, M. Sorel, and S Yu, "All-optical digital logic gates using bistable semiconductor ring lasers," J Opt. Commun., vol.30, pp.190-194, Dec. 2009.

[10] K. Thakulsukanant, B. Li, I. Memon, G. Mezosi, Z. Wang, M. Sorel, and $\mathrm{S} \mathrm{Yu}$, "All-optical label swapping using bistable semiconductor ring laser in an optical switching node", J. Lightw. Technol., vol.20, pp.631-638, Mar. 2009.

[11] J. P. Jiang. Semiconductor laser, Beijing: Publishing House of Electronic Industry, 2000, pp.52-57.

[12] T. Higashi, T. Yamamoto, S. Ogita, and $M$ Kobayashi, "Experimental analysis of termperature dependence of oscillation wavelength in quantum-well FP semiconductor lasers," IEEE J. Quantum Electron., vol.34, pp.1680-1689, Sep.1998.

[13] P W A McIlroy, A. Kurobe, and Y. Uematsu, "Analysis and application of theoretical gain curves to the design of multiquantum-well lasers,” IEEE J. Quantum Electron., vol.QE-21, pp.1958-1963, Dec. 1985.

[14] C Shan, "Temperature's effect on semiconductor laser performance parameter", J. Jilin Normal University, vol.24, pp.95-97, Nov.2003. 